\title{
AN ESTIMATE OF FRÉCHET DISTANCES ON SURFACES OF BOUNDED CURVATURE
}

FOLKE ERIKSSON

1. Introduction. The subject of this paper belongs to the intrinsic geometry of surfaces. By a surface we mean a two-dimensional manifold provided with a Riemannian metric. For our purpose it suffices to consider open, simply connected surfaces. Such a surface $\Phi$ may be defined as a simply connected domain $\omega$ of the $x y$-plane in which there is given a line element

$$
d s^{2}=E d x^{2}+2 F d x d y+G d y^{2},
$$

where the functions $E(x, y), F(x, y), G(x, y)$ defined in $\omega$ satisfy certain conditions of regularity.

Let $\lambda$ and $\mu$ be two point sets on $\Phi$. Their geodesic distance $d(\lambda, \mu)$ is by definition the greatest lower bound of the lengths of all rectifiable curves on $\Phi$ which connect a point of $\lambda$ with a point of $\mu$. The Fréchet distance $D(\lambda, \mu)$ of $\lambda$ and $\mu$ is defined to be the minimum number $D$ such that the geodesic distance from every point of either set to the other set is smaller than or equal to $D$, that is,

$$
D(\lambda, \mu)=\max \left(\sup _{Q \in \mu} d(\lambda, Q), \sup _{P \in \lambda} d(P, \mu)\right) .
$$

Let now $A$ and $B$ be two points on a surface $\Phi$ and $L$ their geodesic distance. Let further $\gamma_{1}$ and $\gamma_{2}$ be two rectifiable curves with lengths $L_{1}$ and $L_{2}$, respectively, which connect $A$ and $B$, and let $D$ denote their Fréchet distance. Then, for surfaces of total (Gaussian) curvature $K \leqq 0$, the following inequality was proved by A. Beurling [2]:

$$
L^{2}+D^{2} \leqq \frac{1}{4}\left(L_{1}+L_{2}\right)^{2} .
$$

Actually Beurling assumes only that the given metric can be written

$$
d s^{2}=e^{2 u(x, y)}\left(d x^{2}+d y^{2}\right),
$$

where $u(x, y)$ is an arbitrary subharmonic function. If $u$ is sufficiently regular, such that the curvature $K$ exists in the usual sense, then this assumption is known to be equivalent to $K \leqq 0$.

Received May 13, 1956. 
Our aim is to prove a similar inequality when an arbitrary upper bound $K_{0}$ of the curvature $K$ is given. In Part $\mathrm{A}$ of the present paper it is assumed that the surface $\Phi$ is "regular", in the sense that the coefficients of the line element possess continuous derivatives of the second order, and that there exists on the surface $\Phi$ a geodesic connecting $A$ and $B$. Then classical methods of differential geometry are applicable and yield for surfaces satisfying $K \leqq K_{0}$ in the cases $K_{0}>0$ and $K_{0}<0$, respectively,

$$
\cos k L \cos k D \geqq \cos \frac{1}{2} k\left(L_{1}+L_{2}\right),
$$

provided that $2 k L<\pi, 2 k L_{1} \leqq \pi, 2 k L_{2} \leqq \pi$, and

$$
\cosh c L \cosh c D \leqq \cosh \frac{1}{2} c\left(L_{1}+L_{2}\right),
$$

where we have put

and

$$
K_{0}=4 k^{2}, k>0, \quad \text { for } K_{0}>0
$$

$$
K_{0}=-4 c^{2}, \quad c>0, \quad \text { for } K_{0}<0 .
$$

In Part $\mathrm{C}$ of the paper the case $K_{\mathbf{0}}<0$ is dealt with under more general assumptions similar to those made by Beurling. Without assuming the existence of a geodesic connecting $A$ and $B$ it is shown, by a method analogous to that developed by Beurling, that (4) still holds. The line element of the surface is supposed to have the form (2), where $u(x, y)$ belongs to a class of continuous functions, called functions of curvature $\leqq K_{0}$, introduced and studied in Part B. The twice differentiable functions of this class are precisely the functions $u$ satisfying the differential inequality

$$
\Delta u \geqq-K_{0} e^{2 u}
$$

which expresses that the curvature of the metric (2) does not exceed $K_{0}$.

The inequalities (1), (3) and (4) imply that every minimizing sequence of curves connecting the points $A$ and $B$, that is, a sequence of curves $\gamma_{i}, i=1,2, \ldots$, whose lengths $L_{i}$ tend to the geodesic distance $L$ of $A$ and $B$, is a Cauchy sequence in the sense that the Fréchet distance $D\left(\gamma_{i}, \gamma_{j}\right)$ tends to zero as $i, j \rightarrow \infty$.

I wish to express my gratitude to Professor Beurling, who directed my attention to this problem, and to Professors Carleson and Fenchel for inspiring discussions and valuable suggestions.

\section{A. Regular surfaces.}

2. Surfaces of constant curvature. We begin by proving the inequalities in the case of a surface of constant curvature $K_{0}$. The intrinsic 
geometry of the surface is then spherical, euclidean, or hyperbolic according as $K_{0}>0, K_{0}=0$, or $K_{0}<0$. Here the geodesic distance between two points equals the length of the (shortest) line segment connecting the points. Although the following results are rather obvious in the euclidean case, we include it for comparison and for the sake of completeness.

Let $\alpha$ denote the line segment connecting the points $A$ and $B$, and let $L$ denote its length, assumed to be smaller than $\pi / 2 k$ in the spherical case. Consider a rectifiable curve $\gamma$ connecting $A$ and $B$ with length $L_{\gamma}(\leqq \pi / 2 k$ in the spherical case). In order to estimate the Fréchet distance

we observe first that

$$
D(\alpha, \gamma)=\max \left(\sup _{Q \in \gamma} d(\alpha, Q), \sup _{P \in \alpha} d(P, \gamma)\right)
$$

$$
D(\alpha, \gamma)=\sup _{Q \in \gamma} d(\alpha, Q) \text {. }
$$

Indeed, let $P$ be an arbitrary point of $\alpha$. The normal to $\alpha$ through $P$ must intersect $\gamma$. Let $Q_{0}$ be one of the points of intersection. Then we have

$$
d(P, \gamma) \leqq d\left(P, Q_{0}\right)=d\left(\alpha, Q_{0}\right) \leqq \sup _{Q \in \gamma} d(\alpha, Q)
$$

since the normal $P Q_{0}$ is the shortest connection between $\alpha$ and $Q_{0}$, and hence

$$
\sup _{P \in \alpha} d(P, \gamma) \leqq \sup _{Q \in \gamma} d(\alpha, Q) .
$$

Consider now the ellipse $\beta$ with foci $A, B$ and major axis $L_{\gamma}$, that is, the locus of the points whose distances from $A$ and $B$ have the sum $L_{\gamma}$. No point $Q$ of $\gamma$ can be outside this ellipse since $L_{\gamma} \geqq d(A, Q)+d(B, Q)$. Hence we have

$$
D(\alpha, \gamma)=\sup _{Q \in \gamma} d(\alpha, Q) \leqq \sup _{R \in \beta} d(\alpha, R) .
$$

We are going to show that the right member of this inequality equals the minor semi-axis $b$ of the ellipse, which is determined by the relations

$$
\begin{aligned}
\cos k L \cos 2 k b & =\cos k L_{\gamma} & & \text { for } \quad K_{0}=4 k^{2}>0, \\
L^{2}+4 b^{2} & =L_{\gamma}^{2} & & \text { for } \quad K_{0}=0, \\
\cosh c L \cosh 2 c b & =\cosh c L_{\gamma} & & \text { for } \quad K_{0}=-4 c^{2}<0 .
\end{aligned}
$$

This statement is equivalent to

$$
d(\alpha, R) \leqq b
$$

for every point $R$ of the ellipse $\beta$. Draw the normals to $\alpha$ through the endpoints $A$ and $B$ and denote their points of intersection with $\beta$ by $A^{\prime}, A^{\prime \prime}$ and $B^{\prime}, B^{\prime \prime}$, respectively. Consider first a point $R$ of the ellipse lying 
outside the strip bounded by the two normals, say beyond $A^{\prime} A A^{\prime \prime}$. Let $S$ be that point of the ray emanating from $A$ and passing through $R$ for which $d(A, S)=d\left(A, A^{\prime}\right)$. In the triangles $A B A^{\prime}$ and $A B S$ we then have $\Varangle B A A^{\prime}<\Varangle B A S$ and hence, by a well-known elementary theorem common to the three geometries, $d\left(B, A^{\prime}\right)<d(B, S)$ and thus

$$
d(A, S)+d(B, S)>d\left(A, A^{\prime}\right)+d\left(B, A^{\prime}\right)=L_{\gamma} .
$$

This means that $S$ is outside the ellipse $\beta$, and this implies

$$
d(A, R)<d(A, S)=d\left(A, A^{\prime}\right) .
$$

Therefore it suffices to consider points $R \in \beta$ belonging to the strip bounded by the normals. Then $d(\alpha, R)=d(N, R)$, where $N$ denotes the foot of the perpendicular from $R$ on $\alpha$. Putting

we have

$$
\begin{gathered}
d(N, R)=h, \quad d(A, N)=a_{1}, \quad d(B, N)=a_{2}, \\
d(A, R)=f_{1}, \quad d(B, R)=f_{2},
\end{gathered}
$$

$$
a_{1}+a_{2}=L, \quad f_{1}+f_{2}=L_{\gamma},
$$

and for $i=1,2$

$$
\begin{aligned}
& \cos 2 k h \cos 2 k a_{i}=\cos 2 k f_{i} \quad \text { if } \quad K_{0}>0 \text {, } \\
& h^{2}+a_{i}^{2}=f_{i}^{2} \quad \text { if } K_{0}=0, \\
& \cosh 2 c h \cosh 2 c a_{i}=\cosh 2 c f_{i} \quad \text { if } \quad K_{0}<0 .
\end{aligned}
$$

It remains to be shown that these relations imply $h \leqq b$. This is easily seen by means of the following lemmas:

LemMA 1. Let $p_{1}, p_{2}, q_{1}, q_{2}$ be non-negative numbers such that $p_{1}+p_{2} \leqq \pi, q_{1} \leqq \pi, q_{2} \leqq \pi$ and $0 \leqq \lambda \leqq 1$. Then

imply

$$
\lambda \cos p_{1}=\cos q_{1}, \quad \lambda \cos p_{2}=\cos q_{2}
$$

$$
\lambda \cos \frac{1}{2}\left(p_{1}+p_{2}\right) \geqq \cos \frac{1}{2}\left(q_{1}+q_{2}\right) .
$$

Lemma 2. Let $p_{1}, p_{2}, q_{1}, q_{2}, l$ be non-negative numbers. Then

imply

$$
l^{2}+{p_{1}}^{2}=q_{1}{ }^{2}, \quad l^{2}+p_{2}^{2}=q_{2}^{2}
$$

$$
l^{2}+\frac{1}{4}\left(p_{1}+p_{2}\right)^{2} \leqq \frac{1}{4}\left(q_{1}+q_{2}\right)^{2} .
$$

Lemma 3. Let $p_{1}, p_{2}, q_{1}, q_{2}$ be non-negative numbers and $\lambda \geqq 1$. Then imply

$$
\lambda \cosh p_{1}=\cosh q_{1}, \quad \lambda \cosh p_{2}=\cosh q_{2}
$$

$$
\lambda \cosh \frac{1}{2}\left(p_{1}+p_{2}\right) \leqq \cosh \frac{1}{2}\left(q_{1}+q_{2}\right) .
$$


Indeed, (8) and Lemma 1 with $p_{i}=2 k a_{i}, q_{i}=2 k f_{i}, \lambda=\cos 2 k h$ yield $\cos k L \cos 2 k h=\cos k\left(a_{1}+a_{2}\right) \cos 2 k h \geqq \cos k\left(f_{1}+f_{2}\right)=\cos k L_{\gamma}$

which, compared with (5), shows that $h \leqq b$. In the same way this follows in the other cases from Lemmas 2 and 3, (6) and (7).

Proofs. Using Cauchy's inequality

$$
\left(x_{1}^{2}+x_{2}^{2}\right)\left(y_{1}^{2}+y_{2}^{2}\right) \geqq\left(x_{1} y_{1}+x_{2} y_{2}\right)^{2},
$$

we obtain in the case of Lemma 2

$$
\begin{aligned}
\left(q_{1}+q_{2}\right)^{2} & =\left(\left(l^{2}+p_{1}{ }^{2}\right)^{\frac{1}{2}}+\left(l^{2}+p_{2}{ }^{2}\right)^{\frac{1}{2}}\right)^{2} \\
& =2 l^{2}+{p_{1}}^{2}+{p_{2}}^{2}+2\left(l^{2}+p_{1}{ }^{2}\right)^{\frac{1}{2}}\left(l^{2}+p_{2}{ }^{2}\right)^{\frac{1}{2}} \\
& \geqq 2 l^{2}+{p_{1}}^{2}+{p_{2}}^{2}+2\left(l^{2}+p_{1} p_{2}\right)=4 l^{2}+\left(p_{1}+p_{2}\right)^{2} .
\end{aligned}
$$

Applying Cauchy's inequality with $x_{1}=y_{1}=\left(1-\lambda^{2}\right)^{\frac{1}{2}}, x_{2}=\lambda \sin p_{1}$, $y_{2}=\lambda \sin p_{2}$, we obtain in the case of Lemma 1

$$
\begin{aligned}
& 2 \cos ^{2} \frac{1}{2}\left(q_{1}+q_{2}\right) \\
& \quad=1+\cos \left(q_{1}+q_{2}\right) \\
& \quad=1+\lambda^{2} \cos p_{1} \cos p_{2}-\left(1-\lambda^{2}+\lambda^{2} \sin ^{2} p_{1}\right)^{\frac{1}{2}}\left(1-\lambda^{2}+\lambda^{2} \sin ^{2} p_{2}\right)^{\frac{1}{2}} \\
& \quad \leqq 1+\lambda^{2} \cos p_{1} \cos p_{2}-\left(1-\lambda^{2}+\lambda^{2} \sin p_{1} \sin p_{2}\right) \\
& \quad=\lambda^{2}\left(1+\cos \left(p_{1}+p_{2}\right)\right)=2 \lambda^{2} \cos ^{2} \frac{1}{2}\left(p_{1}+p_{2}\right) .
\end{aligned}
$$

The proof of Lemma 3 is similar and we omit it.

Returning to our problem, we consider two rectifiable curves $\gamma_{1}, \gamma_{2}$ with lengths $L_{1}, L_{2}$ which connect $A$ and $B$. From what has just been proved it follows that the Fréchet distances $D_{1}=D\left(\alpha, \gamma_{1}\right)$ and $D_{2}=D\left(\alpha, \gamma_{2}\right)$ satisfy the inequalities

$$
D_{1} \leqq b_{1}, \quad D_{2} \leqq b_{2},
$$

where $b_{j}, j=1,2$, according to (5), (6), (7), are determined by

$$
\begin{aligned}
\cos k L \cos 2 k b_{j} & =\cos k L_{j} & & \text { for } K_{0}>0, \\
L^{2}+4 b_{j}^{2} & =L_{j}^{2} & & \text { for } K_{0}=0, \\
\cosh c L \cos 2 c b_{j} & =\cosh c L_{j} & & \text { for } \quad K_{0}<0 .
\end{aligned}
$$

Now it follows immediately from the definition of the Fréchet distance that $D=D\left(\gamma_{1}, \gamma_{2}\right) \leqq D_{1}+D_{2}$. Hence we have

$$
D \leqq b_{1}+b_{2} \text {. }
$$

Eliminating $b_{1}$ and $b_{2}$ by means of (9), (10) and (11), respectively, we finally obtain in the three cases 


$$
\begin{aligned}
& \cos ^{2} k L \cos 2 k D \\
& \quad \geqq \cos k L_{1} \cos k L_{2}-\left(\cos ^{2} k L-\cos ^{2} k L_{1}\right)^{\frac{1}{2}}\left(\cos ^{2} k L-\cos ^{2} k L_{2}\right)^{\frac{1}{2}}, \\
& D \leqq\left(L_{1}^{2}-L^{2}\right)^{\frac{1}{2}}+\left(L_{2}^{2}-L^{2}\right)^{\frac{1}{2}}, \\
& \quad \cosh ^{2} c L \cosh 2 c D \\
& \quad \leqq \cosh c L_{1} \cosh c L_{2}+\left(\cosh ^{2} c L_{1}-\cosh ^{2} c L\right)^{\frac{1}{2}}\left(\cosh ^{2} c L_{2}-\cosh ^{2} c L\right)^{\frac{1}{2}} .
\end{aligned}
$$

In the sequel we shall however be content with the slightly weaker, but simpler inequalities stated in the Introduction. On account of (9), (10) and (11), Lemmas 1, 2 and 3 with

and

$$
\begin{array}{lllll}
p_{1}=2 k b_{1}, & p_{2}=2 k b_{2}, & q_{1}=k L_{1}, & q_{2}=k L_{2}, & \lambda=\cos k L \\
p_{1}=2 b_{1}, & p_{2}=2 b_{2}, & q_{1}=L_{1}, & q_{2}=L_{2}, & l=L
\end{array}
$$

$$
p_{1}=2 c b_{1}, \quad p_{2}=2 c b_{2}, \quad q_{1}=c L_{1}, \quad q_{2}=c L_{2}, \quad \lambda=\cosh c L ;
$$

respectively, yield

$$
\begin{aligned}
\cos k L \cos k\left(b_{1}+b_{2}\right) & \geqq \cos \frac{1}{2} k\left(L_{1}+L_{2}\right), \\
L^{2}+\left(b_{1}+b_{2}\right)^{2} & \leqq \frac{1}{4}\left(L_{1}+L_{2}\right)^{2}, \\
\cosh c L \cosh c\left(b_{1}+b_{2}\right) & \leqq \cosh \frac{1}{2} c\left(L_{1}+L_{2}\right) .
\end{aligned}
$$

Hence, by (12), we obtain the inequalities (3), (1) and (4) for surfaces of constant curvature.

3. An arbitrary regular surface compared with a surface of constant curvature. The restriction on $D$ by (3), (1) and (4) is the stronger the smaller the curvature $K_{\mathbf{0}}$. It is therefore a natural hypothesis that the inequalities obtained for constant curvature will hold a fortiori in the case of variable bounded curvature $K \leqq K_{0}$. This will now be proved.

We consider a surface given by a Riemannian metric

$$
d s^{2}=E d x^{2}+2 F d x d y+G d y^{2}
$$

defined on a simply connected region $\omega$ of the $x y$-plane. The functions $E, F, G$ of $x$ and $y$ are supposed to have continuous partial derivatives of the second order. Then there exists through every point in each direction a unique geodesic (cf. e.g. Eisenhart [5, p. 172]). By $K_{0}$ we denote an upper bound of the curvature $K$.

In $\omega$ we consider a geodesic $\operatorname{arc} A B$ of length $L\left(<\pi / 2 k\right.$ if $\left.K_{0}=4 k^{2}>0\right)$. In a certain subregion $\omega^{*}$ of $\omega$ containing $A B$ we construct another coordinate system in the following way: Through every point $N$ on $A B$ the geodesic normal to $A B$ is drawn. As the coordinates of a point $P$ on this normal we choose the length $u$ of the geodesic arc NP (provided 
with a sign in the usual way) and the length $v$ of the geodesic arc $A N$. The line element takes then the form

$$
d s^{2}=d u^{2}+S(u, v)^{2} d v^{2}
$$

as $F=0$ because the system is orthogonal and $E=1$ because $u$ measures the arc along the curves $v=$ constant. The curvature $K$ is given by the relation

$$
\frac{\partial^{2} S}{\partial u^{2}}+K S=0
$$

(cf. e.g. Darboux, [4, p. 92]). Further the function $S(u, v)$ satisfies the initial conditions

$$
S(0, v)=1, \quad \frac{\partial S}{\partial u}(0, v)=0
$$

because $v$ measures the arc along the curve $u=0$ and because this curve is a geodesic.

In the regions outside the normals to $A B$ through $A$ and $B$ we use geodesic polar coordinates $(r, \theta)$ with $A$ as pole and $(\varrho, \varphi)$ with $B$ as pole. The line elements take here the forms

$$
d s^{2}=d r^{2}+S_{1}(r, \theta)^{2} d \theta^{2},
$$

where

$$
\frac{\partial^{2} S_{1}}{\partial r^{2}}+K S_{1}=0, \quad S_{1}(0, \theta)=0, \quad \frac{\partial S_{1}}{\partial r}(0, \theta)=1
$$

and

$$
d s^{2}=d \varrho^{2}+S_{2}(\varrho, \varphi)^{2} d \varphi^{2},
$$

where

$$
\frac{\partial^{2} S_{2}}{\partial \varrho^{2}}+K S_{2}=0, \quad S_{2}(0, \varphi)=0, \quad \frac{\partial S_{2}}{\partial \varrho}(0, \varphi)=1 .
$$

The region $\omega^{*} \subseteq \omega$, to which we shall confine our considerations, is supposed to be a neighbourhood of the arc $A B$ with the following properties: The geodesic normals to $A B$ together with the rays issuing from $A$ and $B$, which are used in the polar coordinate systems, cover $\omega^{*}$ completely and simply. Each of these normals or rays intersects $\omega^{*}$ in one arc. If $K_{0} \leqq 0$, it follows from well-known theorems that no two of the geodesics in question meet at points different from $A$ and $B$, and $\omega^{*}$ may therefore contain the arc of such a geodesic from $A B$ to the first boundary point of $\omega$ which it contains. In the case $K_{0}>0$ we add the further restriction that the lengths measured from $A B$ of the arcs of the normals and rays contained in $\omega^{*}$ do not exceed $\pi / 4 k$. 
For comparison we introduce in $\omega^{*}$ an auxiliary metric of constant curvature $K_{0}$. This is done by changing the functions $S, S_{1}, S_{2}$ into functions $S^{0}, S_{1}^{0}, S_{2}^{0}$, respectively, which satisfy the same initial conditions but other differential equations, namely

$$
\frac{\partial^{2} S^{0}}{\partial u^{2}}+K_{0} S^{0}=0, \quad \frac{\partial^{2} S_{1}^{0}}{\partial r^{2}}+K_{0} S_{1}^{0}=0, \quad \frac{\partial^{2} S_{2}^{0}}{\partial \varrho^{2}}+K_{0} S_{2}^{0}=0 .
$$

According to a classical result of Sturm [9] (cf. also Bieberbach [3, p. 168-170]) concerning the solutions of differential equations such as (17), (19), (21), it follows from $K \leqq K_{0}$ that

$$
S(u, v) \geqq S^{0}(u, v), \quad S_{1}(r, \theta) \geqq S_{1}^{0}(r, \theta), \quad S_{2}(\varrho, \varphi) \geqq S_{2}^{0}(\varrho, \varphi)
$$

for all points in $\omega^{*}$. In fact, according to Sturm, these inequalities hold generally if $K_{0} \leqq 0$, and in the intervals

thus in $\omega^{*}$.

$$
|u|, r, \varrho \leqq \frac{1}{2} \pi K_{0}^{-\frac{1}{2}} \quad \text { if } \quad K_{0}>0 ，
$$

We denote line elements in the auxiliary metric by $d s^{\mathbf{0}}$. Because of (22) we have $d s \geqq d s^{0}$ for all corresponding line elements in the two metrics.

Let now $\gamma_{j}, j=1,2$, be two arbitrary rectifiable curves in $\omega^{*}$ which connect $A$ and $B$. Let $L_{j}$ and $L_{j}^{0}$ denote their lengths in the original and the auxiliary metries, resp. Then we have

$$
L_{j}^{0} \leqq L_{j} .
$$

On the other hand, the length of the geodesic $A B$ is evidently the same in both metrics:

$$
L^{\mathbf{0}}=L .
$$

Furthermore the Fréchet distance between $\gamma_{j}$ and $A B$ is the same in both metrics :

$$
D_{j}^{0}=D_{j} .
$$

For, the shortest distance to $\gamma_{j}$ from a point $P$ on $A B$ is $\leqq P Q$, if $Q$ denotes a point where $\gamma_{j}$ intersects the normal to $A B$ in $P$; and $P Q$ is the shortest distance from $Q$ to $A B$, since the normals to $A B$ do not intersect in $\omega^{*}$. Thus $D_{j}\left(D_{j}^{0}\right)$ must be the shortest distance to $A B$ from a certain point on $\gamma_{j}$. This distance is situated along one of the normals or rays of our system, and distances along these agree in the two metrics (cf. (16), (18), (20)).

Applying the results of the preceding section to the auxiliary metric, we obtain for the Fréchet distance

$$
D \leqq D_{1}+D_{2}=D_{1}^{0}+D_{2}^{0} \leqq b_{1}+b_{2},
$$


where $b_{j}, j=1,2$, are now determined by (9), (10) or (11) with $L=L^{0}$ and $L_{j}^{0}$ instead of $L_{j}$. Using (23), we therefore obtain the inequalities (3), (1) and (4) from (13), (14) and (15), respectively, which hold with $L_{j}$ replaced by $L_{j}^{0}$. We sum up our results in

TheOREm 1. For the Fréchet distance $D$ between two curves $\gamma_{1}$ and $\gamma_{2}$ with lengths $L_{1}$ and $L_{2}$, which, in the neighbourhood $\omega^{*}$ of a geodesic arc $A B$ with length $L$ on a regular surface, connect the points $A$ and $B$, the following estimates hold:

$$
\cos k L \cos k D \geqq \cos \frac{1}{2} k\left(L_{1}+L_{2}\right),
$$

if the total curvature $K \leqq 4 k^{2}$ and $L<\pi / 2 k, L_{1} \leqq \pi / 2 k, L_{2} \leqq \pi / 2 k$;

if $K \leqq 0$;

$$
L^{2}+D^{2} \leqq \frac{1}{4}\left(L_{1}+L_{2}\right)^{2},
$$

if $K \leqq-4 c^{2}$.

$$
\cosh c L \cosh c D \leqq \cosh \frac{1}{2} c\left(L_{1}+L_{2}\right) \text {, }
$$

\section{B. Functions of bounded curvature.}

4. The definition. Our geometric problem leads to a differential inequality of the form

$$
\Delta u(z) \geqq-x e^{2 u(z)}, \quad z=x+i y .
$$

We wish to extend the class of functions satisfying (24) such as to include functions which do not have the derivatives in question, in the same way as subharmonic functions correspond to the inequality $\Delta u \geqq 0$. However, we restrict ourselves to continuous functions. We use the notations for mean values current in the theory of subharmonic functions (cf. e.g. Radó [7]):

$$
\begin{aligned}
& L\left(u, z_{0}, r\right)=\frac{1}{2 \pi} \int_{0}^{2 \pi} u\left(z_{0}+r e^{i \theta}\right) d \theta, \\
& A\left(u, z_{0}, r\right)=\frac{1}{\pi r^{2}} \int_{0}^{r} \int_{0}^{2 \pi} u\left(z_{0}+\varrho e^{i \theta}\right) \varrho d \varrho d \theta .
\end{aligned}
$$

Integrating (24) over a circular disk about $z_{0}$, one finds by Gauss' theorem

$$
\frac{\partial}{\partial r} L\left(u, z_{0}, r\right) \geqq-\frac{1}{2} \varkappa r A\left(e^{2 u}, z_{0}, r\right) .
$$

Integration of (25) between 0 and $r$ yields

$$
L\left(u, z_{0}, r\right)-L\left(u, z_{0}, 0\right) \geqq-\frac{1}{2} \varkappa \int_{0}^{r} \varrho A\left(e^{2 u}, z_{0}, \varrho\right) d \varrho
$$


or

$$
L\left(u, z_{0}, r\right)-u\left(z_{0}\right) \geqq-\frac{1}{2} \varkappa E\left(u, z_{0}, r\right) ;
$$

with the notation

$$
E\left(u, z_{0}, r\right)=\int_{0}^{r} \varrho A\left(e^{2 u}, z_{0}, \varrho\right) d \varrho .
$$

Thus, if we disregard a factor depending on $r, E\left(u, z_{0}, r\right)$ is a mean value of $e^{2 u}$.

By means of the inequality (26) we now define for every real $x$ the class $C(\varkappa)$ of functions of curvature $\leqq \varkappa$ without using derivatives:

Definition. $u \in C(x)$ in a region $\omega$ if and only if 1) $u(z)$ is continuous in $\omega$,

2) $u(z)$ satisfies (26) for every $z_{0}$ in $\omega$ and for all sufficiently small $r$.

Reversing the argument leading to (26) it is seen that if $u(z)$ has continuous partial derivatives of the second order, $u(z) \in C(x)$ if and only if it satisfies (24). In particular, the solutions of the differential equation $\Delta u=-x e^{2 u}$ satisfy (26) with the sign of equality.

For $\varkappa=0$ we get the subharmonic functions, and if $u \in C(x)$ for a $x<0$, then $u$ is a fortiori subharmonic.

\section{Comparison with solutions of $\Delta u=-x e^{2 u}$.}

Lemma 4. Suppose that $u(z) \in C(\varkappa), \varkappa \leqq 0$, and that $v(z)$ satisfies

$$
\Delta v=-x e^{2 v}
$$

in a region $\omega$. Suppose further that $u(z)$ and $v(z)$ are continuous in the closure of $\omega$ and that $u(z) \leqq v(z)$ on the boundary of $\omega$.

Then $u(z) \leqq v(z)$ holds in $\omega$.

Proof. Sappose $u>v$ on a certain point set $S$ of $\omega$. Because of the continuity, $S$ is an open set. For a $z_{0}$, which together with a neighbourhood of radius $r$ belongs to $S$, we get from (26)

$$
\begin{aligned}
& u\left(z_{0}\right) \leqq L\left(u, z_{0}, r\right)+\frac{1}{2} \varkappa E\left(u, z_{0}, r\right) \leqq L\left(u, z_{0}, r\right)+\frac{1}{2} \varkappa E\left(v, z_{0}, r\right) ; \\
& v\left(z_{0}\right)=L\left(v, z_{0}, r\right)+\frac{1}{2} \varkappa E\left(v, z_{0}, r\right) .
\end{aligned}
$$

Subtraction gives

$$
u\left(z_{0}\right)-v\left(z_{0}\right) \leqq L\left(u, z_{0}, r\right)-L\left(v, z_{0}, r\right)=L\left(u-v, z_{0}, r\right) .
$$

Hence $u-v$ would be subharmonic in $S$. But on the boundary of $S$ $u-v \leqq 0$. Thus our assumption $u-v>0$ in $S$ contradicts the maximum principle. 
6. Comparison with solutions of $\Delta v=-x e^{2 u}$. Lemma 4 does not hold in the case $x>0$, but another method of comparison is applicable for all $x$ :

Lemma 5. Suppose that $u(z) \in C(x)$ and that $v(z)$ is a solution of

$$
\Delta v(z)=-x e^{2 u(z)}
$$

in $\omega$. Suppose further that $u(z)$ and $v(z)$ are continuous in the closure of $\omega$ and that $u(z) \leqq v(z)$ on the boundary of $\omega$. Then $u(z) \leqq v(z)$ holds in $\omega$.

Proof. (26) and integration of (28) yield

Subtraction gives

$$
\begin{aligned}
& u\left(z_{0}\right) \leqq L\left(u, z_{0}, r\right)+\frac{1}{2} \varkappa E\left(u, z_{0}, r\right), \\
& v\left(z_{0}\right)=L\left(v, z_{0}, r\right)+\frac{1}{2} \varkappa E\left(u, z_{0}, r\right) .
\end{aligned}
$$

$$
u\left(z_{0}\right)-v\left(z_{0}\right) \leqq L\left(u, z_{0}, r\right)-L\left(v, z_{0}, r\right)=L\left(u-v, z_{0}, r\right),
$$

that is, $u-v$ is subharmonic (as $z_{0}, r$ are arbitrary). According to the maximum principle, $u-v \leqq 0$ in $\omega$ since $u-v \leqq 0$ on the boundary of $\omega$.

Lemma 6. Suppose that $u(z)$ is continuous in $\omega$ and that, for every circle $C$ in $\omega, u(z) \leqq v_{C}(z)$ holds in $C$ if $v_{C}(z)$ is the solution of (28) which equals $u(z)$ on the boundary of $C$. Then $u(z) \in C(x)$ in $\omega$.

Proof. Considering an arbitrary $z_{0}$ with a neighbourhood of radius $r$ in $\omega$, we have, if $v(z)$ satisfies (28) and $v(z)=u(z)$ for $z=z_{0}+r e^{i \theta}, 0 \leqq \theta \leqq 2 \pi$,

$$
L\left(u, z_{0}, r\right)=L\left(v, z_{0}, r\right)=v\left(z_{0}\right)+\frac{1}{2} \varkappa E\left(u, z_{0}, r\right) \geqq u\left(z_{0}\right)+\frac{1}{2} \varkappa E\left(u, z_{0}, r\right) .
$$

Hence $u(z)$ satisfies (26), and this holds for every circle in $\omega$ since the boundary value problem of (28) is uniquely solvable (Picard [6, chap.III]).

\section{Conformal mapping.}

Lemma 7. If $w=f(z)$ maps a region $\omega$ of the $z$-plane conformally onto $\omega^{\prime}$ in the w-plane, and if $u(w) \in C(x)$ in $\omega^{\prime}$, then the function

in $\omega$.

$$
u(f(z))+\log \left|f^{\prime}(z)\right| \in C(\varkappa)
$$

Proof. This is a consequence of the simple and well-known formula

$$
\Delta_{z} u=\left|f^{\prime}(z)\right|^{2} \Delta_{w} u,
$$

where $\Delta_{z}$ and $\Delta_{w}$ denote the Laplacians in the $z$ - and $w$-planes, respectively. 
Let $v(z)$ be a solution of the equation

$$
\Delta v(z)=-x e^{2 u(f(z))}\left|f^{\prime}(z)\right|^{2}
$$

with the same boundary values as $u(f(z))+\log \left|f^{\prime}(z)\right|$ on a circle $C$ in the $z$-plane. Then the function of $w$

satisfies the equation

$$
\varphi(w)=v(z)-\log \left|f^{\prime}(z)\right|
$$

$$
\Delta \varphi(w)=-\varkappa e^{2 u(w)}
$$

because $\log \left|f^{\prime}(z)\right|$ is harmonic in $\omega$ and because of $(29)$. Now $\varphi(w)$ equals $u(w)$ on the boundary of the image $C^{\prime}$ of $C$ under the mapping $w=f(z)$. According to Lemma 5 we thus have $u(w) \leqq \varphi(w)$ within $C^{\prime}$. Expressing this in terms of $z$ we find

$$
u(z)+\log \left|f^{\prime}(z)\right| \leqq v(z) \quad \text { in } C .
$$

But as the circle $C$ is arbitrary, it follows from Lemma 6 that

in $\omega$.

$$
u(z)+\log \left|f^{\prime}(z)\right| \in C(x)
$$

\section{A lemma on a mean value.}

Lemma 8. If $u \in C(\varkappa), v \in C(\varkappa), x \leqq 0$, and the function $w$ is defined by

$$
e^{w}=\frac{1}{2}\left(e^{u}+e^{v}\right) \text {, }
$$

then $w \in C(x)$.

Proof. If the derivatives exist, we find by calculating $\Delta w$

$$
\begin{aligned}
\Delta w & =\frac{1}{e^{u}+e^{v}}\left(e^{u} \Delta u+e^{v} \Delta v\right)+\frac{e^{u+v}}{\left(e^{u}+e^{v}\right)^{2}}\left\{\left(\frac{\partial u}{\partial x}-\frac{\partial v}{\partial x}\right)^{2}+\left(\frac{\partial u}{\partial y}-\frac{\partial v}{\partial y}\right)^{2}\right\} \\
& \geqq \frac{e^{u} \Delta u+e^{v} \Delta v}{e^{u}+e^{v}} \geqq \frac{-\varkappa\left(e^{3 u}+e^{3 v}\right)}{e^{u}+e^{v}}=-x\left(e^{2 u}-e^{u+v}+e^{2 v}\right) \\
& =-x\left\{\frac{3}{4}\left(e^{u}-e^{v}\right)^{2}+\frac{1}{4}\left(e^{u}+e^{v}\right)^{2}\right\} \geqq-\frac{1}{4} x\left(e^{u}+e^{v}\right)^{2}=-x e^{2 w} .
\end{aligned}
$$

This gives a hint for the general proof: As $\Delta u$ and $\Delta v$ enter multiplied by $e^{u}$ and $e^{v}$, resp., the inequalities (26) for $u$ and $v$ ought to be multiplied by $e^{u}$ and $e^{v}$, resp., before adding them in order to prove (26) for $w$. It suffices to do this for small values of $r$. For the sake of simplicity we omit $z_{0}$ and $r$ in the mean value notations $L$ and $E$ and the argument $z_{0}$ in the functions. Multiplying (26) for $u$ and $v$ by $e^{u}$ and $e^{v}$, respectively, and adding, we obtain

$$
e^{u}(L(u)-u)+e^{v}(L(v)-v) \geqq-\frac{1}{2} \varkappa\left(e^{u} E(u)+e^{v} E(v)\right) .
$$


In order to show that the right member of (31) is greater than or equal to $-x e^{w} E(w)$, we begin by estimating

$$
\begin{aligned}
& E(w)=\int_{0}^{r} \varrho A\left(e^{2 w}, z_{0}, \varrho\right) d \varrho=\int_{0}^{r} \varrho A\left(\frac{1}{4}\left(e^{u}+e^{v}\right)^{2}, z_{0}, \varrho\right) d \varrho \\
& \leqq \int_{0}^{r} \varrho A\left(\frac{1}{2} e^{2 u}+\frac{1}{2} e^{2 v}, z_{0}, \varrho\right) d \varrho=\frac{1}{2}[E(u)+E(v)] .
\end{aligned}
$$

This gives

$$
\begin{aligned}
e^{w} E(w) & =\frac{1}{2}\left(e^{u}+e^{v}\right) E(w) \leqq \frac{1}{4}\left(e^{u}+e^{v}\right)[E(u)+E(v)] \\
& =\frac{1}{2}\left[e^{u} E(u)+e^{v} E(v)\right]-\frac{1}{4}\left(e^{u}-e^{v}\right)[E(u)-E(v)] \leqq \frac{1}{2}\left[e^{u} E(u)+e^{v} E(v)\right] ;
\end{aligned}
$$

for, if $e^{u}-e^{v} \neq 0$, then $e^{u}-e^{v}$ and $E(u)-E(v)$ have the same sign for small $r$ because of the continuity. Applying this result to the right member of $(31)$, we get

$$
e^{u}(L(u)-u)+e^{v}(L(v)-v) \geqq-x e^{w} E(w) .
$$

In order to estimate $L(w)-w$, we apply Taylor's formula to the function $w(u, v)=\log \frac{1}{2}\left(e^{u}+e^{v}\right)$ :

$$
\begin{aligned}
\log \frac{1}{2}\left(e^{u+d u}+e^{v+d v}\right)-\log \frac{1}{2}\left(e^{u}+e^{v}\right) \\
=\frac{e^{u} d u+e^{v} d v}{e^{u}+e^{v}}+\frac{e^{u+\theta d u+v+\theta d v}}{2\left(e^{u+\theta d u}+e^{v+\theta d v}\right)^{2}}(d u-d v)^{2} \geqq \frac{e^{u} d u+e^{v} d v}{e^{u}+e^{v}} .
\end{aligned}
$$

Using this, we obtain

$$
\begin{aligned}
L(w)-w & =L\left(\log \frac{1}{2}\left(e^{u}+e^{v}\right)\right)-\log \frac{1}{2}\left(e^{u}+e^{v}\right) \\
& =\frac{1}{2 \pi} \int_{0}^{2 \pi}\left\{\log \frac{1}{2}\left(e^{u\left(z_{0}+r e^{i \theta}\right)}+e^{v\left(z_{0}+r e^{i \theta}\right)}\right)-\log \frac{1}{2}\left(e^{u\left(z_{0}\right)}+e^{v\left(z_{0}\right)}\right)\right\} d \theta \\
& \geqq \frac{1}{2 \pi} \int_{0}^{2 \pi}\left\{\frac{e^{u\left(z_{0}\right)}\left[u\left(z_{0}+r e^{i \theta}\right)-u\left(z_{0}\right)\right]+e^{v\left(z_{0}\right)}\left[v\left(z_{0}+r e^{i \theta}\right)-v\left(z_{0}\right)\right]}{e^{u\left(z_{0}\right)}+e^{v\left(z_{0}\right)}}\right\} d \theta \\
& =\frac{e^{u}}{e^{u}+e^{v}}[L(u)-u]+\frac{e^{v}}{e^{u}+e^{v}}[L(v)-v],
\end{aligned}
$$

hence, multiplying by $e^{u}+e^{v}=2 e^{w}$,

$$
2 e^{w}[L(w)-w] \geqq e^{u}[L(u)-u]+e^{v}[L(v)-v] .
$$

Applying this result to the left member of (32), we find 


$$
L(w)-w \geqq-\frac{1}{2} \varkappa E(w),
$$

that is (26) for the function $w$. This completes the proof of Lemma 8 .

(30) defines a mean value of two functions, for which we have stated and proved Lemma 8. However, an analogous mean value of $n$ functions may be defined by

and here holds

$$
e^{w}=\frac{1}{n} \sum_{\nu=1}^{n} e^{u_{\nu}}
$$

LEMMA 9. If the functions $u_{\nu}$ in (33) are of curvature $\leqq \varkappa \leqq 0$, so is $w$.

Lemma 9 may be proved by the well-known method of approximating $1 / n$ by numbers of the form $k / 2^{\nu}$. As we need the lemma only for $n=4$, we are content to observe that this case can be settled by repeated application of Lemma 8 .

\section{General treatment of the case $K_{0}<0$.}

9. Generalisation of the notion of bounded curvature. We turn now to the generalisation of our results from Part A by means of the tools developed in Part B. Following Beurling [2], we consider a surface, given by a Riemannian metric

$$
d s=e^{u(z)}|d z|
$$

defined in a region $\omega$ of the complex $z$-plane. If $u(z)$ has partial derivatives of the second order, the total curvature of the metric (34) is

$$
K=-e^{-2 u} \Delta u \text {. }
$$

An upper bound for the curvature, $K \leqq K_{0}$, then leads to the condition

$$
\Delta u \geqq-K_{0} e^{2 u},
$$

that is, the function $u(z)$ shall be of curvature $\leqq K_{0}$.

If the derivatives do not exist, the curvature is not defined. But we say, by definition, that the metric (34) is of curvature $\leqq K_{0}$ if and only if the function $u(z)$ is of curvature $\leqq K_{0}$.

A metric of bounded curvature in this sense is also of bounded curvature in the sense of A. D. Alexandrow ([1, p. 493]). According to a result of J. G. Reschetnjak [8] (cf. [1, p. 503]), a metric (34) is of bounded curvature in Alexandrow's sense, if and only if $u(z)$ is the difference of two subharmonic functions. If $u \in C\left(K_{0}\right), K_{0} \leqq 0$, the function $u$ itself 
is subharmonic. And if $K_{0}>0$, we can write $u=u_{1}-u_{2}$, using the function $v(z)$ of Lemma 5 and putting $u_{1}=u-v, u_{2}=-v$. Then $u_{1}$ and $u_{2}$ are subharmonic (cf. Lemma 5).

10. A theorem on conformal rhombs. For surfaces of curvature $\leqq K_{0}<0$ in the generalised sense of Section 9, we shall first prove a theorem analogous to Beurling's Theorem II in [2]. Our proof will be completely analogous to that of Beurling.

Let $\Omega$ be a simply connected region of the complex plane bounded by a rectifiable Jordan curve, and let $A, B$ be two distinct boundary points of $\Omega$. A curve $\alpha$ in $\Omega$, connecting $A$ with $B$ and dividing $\Omega$ into two parts $\Omega_{1}$ and $\Omega_{2}$, is called a line of symmetry of $\Omega$ if there exists a conformal mapping of $\Omega$ onto itself which leaves every point of $\alpha$ invariant and maps $\Omega_{1}$ onto $\Omega_{2}$ and $\Omega_{2}$ onto $\Omega_{1}$. The configuration $\Omega_{\alpha, \beta}$ formed by the region $\Omega$ and two of its lines of symmetry, $\alpha$ and $\beta$, which intersect at right angles, is called a conformal rhomb. Assuming that the metric (34) is defined in the closure of $\Omega$, we denote the lengths of $\alpha$ and $\beta$ by $a$ and $b$, resp., and the perimeter of $\Omega$ by $p$. We are going to prove

TheORem 2. For a conformal rhomb $\Omega_{\alpha, \beta}$ with a metric (34) of curvature $\leqq K_{0}=-4 c^{2}$, the inequality

$$
\cosh c a \cosh c b \leqq \cosh \frac{1}{2} c p
$$

holds.

Let $T_{1}$ and $T_{2}$ be the conformal transformations of $\Omega$ onto itself which correspond to the lines of symmetry $\alpha$ and $\beta$, respectively. If we denote by $T_{0}$ the identical transformation and by $T_{3}$ the transformation $T_{1} T_{2}$, the transformations $T_{0}, T_{1}, T_{2}$ and $T_{3}$ form a group $G$. A metric (34) is called symmetric with respect to $G$ if

$$
e^{u(z)}|d z|=e^{u\left(T_{\nu} z\right)}\left|d T_{v} z\right|, \quad v=0,1,2,3 .
$$

If the metric is not symmetric, we define a symmetric metric by

$$
d s_{s}=e^{u_{s}(z)}|d z|=\frac{1}{4} \sum_{\nu=0}^{3} e^{u\left(T_{\nu} z\right)}\left|d T_{\nu} z\right| .
$$

The lengths of $\alpha, \beta$ and the perimeter of $\Omega$ are not altered by the symmetrisation because every transformation $T_{v}$ maps $\alpha, \beta$ and the boundary of $\Omega$ onto themselves. Therefore it is sufficient to prove (35) for the metric (36), which is also of curvature $\leqq-4 c^{2}$. In fact, according to $(36), u_{s}(z)$ is defined by 


$$
e^{u_{s}(z)}=\frac{1}{4} \sum_{\nu=0}^{3} e^{u\left(T_{\nu} z\right)+\log \left|d T_{\nu} z / d z\right|} .
$$

Since $u(z) \in C\left(-4 c^{2}\right)$, it follows from Lemma 7 that the functions $u\left(T_{v} z\right)+\log \left|d T_{v} z / d z\right| \in C\left(-4 c^{2}\right)$. Then it follows from Lemma 9 that $u_{s}(z) \in C\left(-4 c^{2}\right)$. Thus it remains to prove (35) for a symmetric metric of curvature $\leqq-4 c^{2}$.

Suppose first that the metric (36) is of constant curvature $-4 c^{2}$. In this case we may suppose that $\Omega$ is embedded in the hyperbolic plane. As the metric is symmetric, $\alpha$ and $\beta$ must be geodesics. Suppose e.g. that the geodesic $A B$ were $\alpha^{\prime} \neq \alpha$. Then the curve $T_{1} \alpha^{\prime}$ (symmetric to $\alpha^{\prime}$ with respect to $\alpha$ ) would have the same length as $\alpha^{\prime}$. This would, however, contradict the uniqueness of geodesics in the hyperbolic plane. Furthermore the geodesics $\alpha$ and $\beta$ are orthogonal in the metric (36) because of the symmetry.

Now, let $C$ be an endpoint of $\beta$. Then the perimeter $p$ of $\Omega$ is at least 4 times the length $l$ of the geodesic $A C$, and the latter can be determined by the cosine theorem of hyperbolic geometry:

$$
\cosh 2 c l=\cosh c a \cosh c b .
$$

Since $p \geqq 4 l$, we obtain (35).

When the metric (36) is not of constant curvature, we introduce for comparison an auxiliary metric in $\Omega$ putting

$$
d s^{\prime}=e^{u^{\prime}(z)}|d z|,
$$

where $u^{\prime}(z)$ satisfies

$$
\Delta u^{\prime}=4 c^{2} e^{2 u^{\prime}},
$$

and has the same boundary values as $u_{s}(z)$. The metric (37) is of constant curvature $-4 c^{2}$, and furthermore it is symmetric. For otherwise the functions $u^{\prime}\left(T_{v} z\right)+\log \left|d T_{v} z / d z\right|, v=0,1,2,3$, would be distinct solutions of (38) with the same boundary values, contrary to the fact that the solution of (38) with given boundary values is uniquely determined (cf. e.g. Picard [6]).

Thus our previous reasoning holds for the metric (37). If we denote the lengths of $\alpha, \beta$ and the perimeter of $\Omega$ in the metric (37) by $a^{\prime}, b^{\prime}$ and $p^{\prime}$, we therefore have

$$
\cosh c a^{\prime} \cosh c b^{\prime} \leqq \cosh \frac{1}{2} c p^{\prime} .
$$

According to Lemma 4, we have $u_{s}(z) \leqq u^{\prime}(z)$ in $\Omega$. Hence it follows that $a \leqq a^{\prime}, b \leqq b^{\prime}$, while $p=p^{\prime}$. Combining this with (39), we obtain (35), and Theorem 2 is proved. 
11. Application to our problem. We now return to the original problem posed in Section 1. Suppose first that the curves $\gamma_{1}$ and $\gamma_{2}$, which connect the points $A$ and $B$, bound a connected region $\Omega$. Let $\alpha$ be the line of symmetry of $\Omega$ which connects $A$ and $B$, and let $\beta$ be an arbitrary line of symmetry orthogonal to $\alpha$. Denoting the lengths of $\alpha$ and $\beta$ by $a$ and $b$, Theorem 2 yields for the conformal rhomb $\Omega_{\alpha, \beta}$, if the curvature is $\leqq-4 c^{2}$,

$$
\cosh c a \cosh c b \leqq \cosh \frac{1}{2} c\left(L_{1}+L_{2}\right) .
$$

From the definition of the geodesic distance we have $L \leqq a$; and when $\beta$ varies, its endpoints describe the whole curves $\gamma_{1}$ and $\gamma_{2}$. Hence, for the Fréchet distance between $\gamma_{1}$ and $\gamma_{2}$ we have $D \leqq \sup b$; for every point of $\gamma_{1}\left(\gamma_{2}\right)$ is connected with a point of $\gamma_{2}\left(\gamma_{1}\right)$ by one of the curves $\beta$. From (40) we then conclude a fortiori

$$
\cosh c L \cosh c D \leqq \cosh \frac{1}{2} c\left(L_{1}+L_{2}\right) \text {. }
$$

It remains to prove (4) when the region bounded by $\gamma_{1}$ and $\gamma_{2}$ is not connected. Then it consists of finitely or infinitely many connected regions. We consider one of these regions $\Omega^{\prime}$ bounded by subarcs $\gamma_{1}^{\prime}$ and $\gamma_{2}{ }^{\prime}$ of $\gamma_{1}$ and $\gamma_{2}$. We denote the lengths of these subarcs by $l_{1}$ and $l_{2}$, the Fréchet distance between $\gamma_{1}{ }^{\prime}$ and $\gamma_{2}^{\prime}$ by $d$, and the geodesic distance between the endpoints of $\gamma_{1}^{\prime}$ and $\gamma_{2}^{\prime}$ by $l$. For the region $\Omega^{\prime},(4)$ holds, that is,

$$
\cosh c d \leqq \frac{\cosh \frac{1}{2} c\left(l_{1}+l_{2}\right)}{\cosh c l} .
$$

To the right member of (41) we now apply

Lemma 10. If $A>B>0, A>a>b>0$ and $A-a \geqq B-b$, then

$$
\frac{\cosh A}{\cosh B}>\frac{\cosh a}{\cosh b} \text {. }
$$

The quantities $\frac{1}{2} c\left(L_{1}+L_{2}\right), c L, \frac{1}{2} c\left(l_{1}+l_{2}\right), c l$ satisfy the conditions on $A, B, a, b$, respectively, in the lemma. The last condition

$$
L-l \leqq \frac{1}{2}\left(L_{1}+L_{2}\right)-\frac{1}{2}\left(l_{1}+l_{2}\right)
$$

is satisfied because even

$$
L \leqq l+\min \left(L_{1}-l_{1}, L_{2}-l_{2}\right) .
$$

Thus from (41) we obtain

$$
\cosh c d \leqq \frac{\cosh \frac{1}{2} c\left(L_{1}+L_{2}\right)}{\cosh c L} .
$$


Obviously, the Fréchet distance $D$ between $\gamma_{1}$ and $\gamma_{2}$ cannot be greater than the least upper bound of $d$ for all different partial $\operatorname{arcs} \gamma_{1}^{\prime}, \gamma_{2}^{\prime}$. As (42) holds for all these $d$, it follows that

$$
\cosh c D \leqq \frac{\cosh \frac{1}{2} c\left(L_{1}+L_{2}\right)}{\cosh c L},
$$

which is (4). Thereby our problem is solved for $K_{0}<0$. We formulate the result:

Theorem 3. In a metric (34) of curvature $\leqq-4 c^{2}$ in the generalised sense of Section 9, the quantities $D, L, L_{1}$ and $L_{2}$ defined in Section 1 satisfy the inequality

$$
\cosh c L \cosh c D \leqq \cosh \frac{1}{2} c\left(L_{1}+L_{2}\right) .
$$

It remains only to prove Lemma 10 .

Proof of Lemma 10. Putting $f(t)=a+(A-a) t$ and $g(t)=b+(B-b) t$, we have $f(t)>g(t), f^{\prime}(t) \geqq g^{\prime}(t)$ for $t \geqq 0$. The function

$$
F(t)=\frac{\cosh f(t)}{\cosh g(t)}
$$

increases for $t \geqq 0$. Indeed,

$$
F^{\prime}(t)=\frac{\left(\cosh g \sinh f f^{\prime}-\cosh f \sinh g g^{\prime}\right)}{\cosh ^{2} g}>0 ;
$$

for $f^{\prime}>0, f^{\prime} \geqq g^{\prime}$ and $\cosh g \sinh f>\cosh f \sinh g>0$ since

$$
\cosh g \sinh f-\cosh f \sinh g=\sinh (f-g)>0 .
$$

Hence we have

$$
\cosh A / \cosh B=F(1)>F(0)=\cosh a / \cosh b .
$$

\section{REFERENCES}

1. A. D. Alexandrow, Die innere Geometrie der konvexen Flächen, Berlin, 1955.

2. A. Beurling, Sur la géométrie métrique des surfaces à courbure totale $\leqq 0$, Medd. Lunds Univ. Mat. Sem., Supplementbd. tillägnat Marcel Riesz (1952), 7-11.

3. L. Bieberbach, Theorie der Differentialgleichungen, 3. Aufl., Berlin, 1930.

4. G. Darboux, Leçons sur la théorie générale des surfaces, III, Paris, 1894.

5. L. P. Eisenhart, An introduction to differential geometry, Princeton, 1940. 
6. É. Picard, Mémoire sur la théorie des équations aux dérivées partielles, J. Math. Pures Appl. (4) 6 (1890), 145-210.

7. T. Radó, Subharmonic functions (Ergebn. Math. 5,1), Berlin, 1937.

8. J. G. Reschetnjak, Isothermal coordinates in manifolds of bounded curvature, Doklady Akad. Nauk SSSR (N. S.) 64 (1954), 631-634. (In Russian.)

9. C. Sturm, Sur les équations différentielles linéaires du second ordre, J. Math. Pures Appl. (1) 1 (1836), 106-186.

UNIVERSITY OF UPPSALA, SWEDEN 